\title{
Innovación por el Usuario en la Construcción y el Diseño Digital: La Relación Entre Herramientas BIM Standard y Requerimientos Específicos de los Usuarios
}

\author{
User Innovation in Digital Design and Construction: The Relation Between Standard BIM Tools and \\ Specific User Requirements
}

\author{
Carolina Soto Ogueta \\ Chile \\ carolinasotoo@yahoo.com
}

\begin{abstract}
BIM tools are being adopted in many different countries, in a wide range of types of projects, and by professionals from different disciplines. Consequently, the requirements of its users are becoming heterogeneous, hindering the development of BIM tools that can satisfy all of them. Utilizing the user innovation theory as the framework of analysis, the paper examines whether and how BIM users are adapting the technology to their requirements. Through the study of eight cases from different contexts of use, the work analyzes the process of user innovation in BIM with two main objectives: first, to recognize whether there is that type of innovation, and second, to understand how it is developed.
\end{abstract}

Keywords: BIM; User Innovation; Adaptation; Customization; Context of use.

\section{Introducción}

Las herramientas BIM (Building Information Modeling) permiten generar modelos que no sólo contienen información acerca de la forma de los proyectos, sino también de sus materiales, componentes, estructura, costos y organización espacial, entre otros datos. Estas tecnologías están siendo adoptadas en diversos países, por profesionales de diferentes disciplinas, para desarrollar un amplio rango de tipos de proyectos. Es decir, las herramientas BIM están siendo aplicadas en diversos contextos de uso, dado que son usadas por una población con características diferenciadas "en un rango de entornos técnicos, físicos, y sociales u organizacionales que pueden afectar su uso" (Maguire, 2001, p. 453). Debido a estos dos factores: la complejidad de la información que se puede introducir en un modelo BIM y los diversos contextos de uso donde se está utilizando, los requerimientos de sus usuarios se vuelven cada vez más heterogéneos, dificultando el desarrollo de herramientas que puedan satisfacerlos todos. En cambio, las herramientas son desarrolladas para satisfacer las necesidades más estándares y genéricas de la industria de la Arquitectura, Ingeniería y Construcción.

La necesidad de salvar la brecha entre herramientas BIM estándares y necesidades específicas de los usuarios genera oportunidades para la innovación. La hipótesis de la investigación es que los usuarios de BIM, siguiendo lo descrito por la literatura de innovación por el usuario, están desarrollando nuevos procesos, plug-ins y componentes digitales para adaptar las herramientas a sus requerimientos, generando una relación dialéctica entre tecnologías estándar y requerimientos específicos de los usuarios. Estas innovaciones bottom-up tienen el potencial de prevenir la excesiva estandarización formal que puede resultar del uso de herramientas genéricas.

\section{Metodología}

El artículo presenta la investigación realizada por el autor entre 2011 y 2012 en el marco de su tesis de Magister en el Design and Computation Group del Massachusetts Institute of Technology. Utilizando la teoría de la innovación por el usuario como marco de análisis, el trabajo examina cómo los usuarios de BIM están adaptando las herramientas estándares para responder a sus necesidades específicas. A través del estudio de ocho casos provenientes de distintos contextos de uso, se analiza el proceso de innovación por el usuario en BIM, con dos objetivos: primero, reconocer si existe o no este tipo de innovación en el caso de las herramientas BIM y segundo, entender cómo se desarrolla esa innovación. Finalmente, el trabajo extrae patrones y examina si éstos coinciden con lo expuesto por la teoría de innovación por el usuario.

\section{Teoría de Innovación por el usuario}

Según Von Hippel (2005) la innovación por el usuario es la modificación de productos y tecnologías existentes o el desarrollo de nuevos productos, por parte de sus usuarios, entendiendo a estos últimos como individuos o empresas. Una de las razones principales para la aparición de innovación por el usuario es la asimetría de la información, esto es la diferencia entre el conocimiento de los fabricantes y de los usuarios de un producto. 
Mientras los fabricantes tienen un amplio conocimiento de las necesidades generales del mercado y de cómo abordar estas soluciones a nivel global, los usuarios en cambio tienen conocimiento sobre sus necesidades específicas, la manera en la cual usan las herramientas y su contexto de uso particular (Von Hippel, 2005). Muchas veces el conocimiento que los usuarios tienen acerca de su uso de un cierto producto es tácito (Polanyi, 1958) y local (Luthje et. al., 2005), por lo cual la transferencia de ese conocimiento es costosa y difícil de lograr. Consecuentemente, esta información tiende a quedarse en su lugar de origen (Von Hippel, 1994), fomentando un cambio en la locación de la innovación, desde el productor al usuario.

Con el fin de mantenerse competitivos en este contexto y dado que satisfacer mercados individuales es económicamente inviable, los fabricantes buscan otros métodos para abordar las necesidades heterogéneas de los usuarios, traduciéndose esto en apoyo directo e indirecto a la innovación por parte de los usuarios. Dos de las principales estrategias de apoyo indirecto a la innovación son: el desarrollo de kits de herramientas para los usuarios (user toolkits) y el fomento de las comunidades de usuarios. Los kits consisten en sets de herramientas que permiten a los usuarios diseñar sus productos o modificar los existentes, generar prototipos y testearlos (Von Hippel, 2005; Thomke \& Von Hippel, 2002). En el caso de los software estos sets de herramientas corresponden a los SDK (Software Development Kit) que posibilitan el desarrollo de plug-ins para perfeccionar el producto original y permitir al usuario desempeñar ciertas tareas específicas no contempladas en el software. La mayoría de las herramientas BIM existentes contiene SDK y abren el acceso a sus API (Application Programming Interface) permitiendo a los usuarios programar sus propias funciones sobre el software original.

Las comunidades de usuarios, por su parte, generan comunicación y transferencia de conocimiento entre usuarios, promoviendo el uso de las herramientas o productos y resolviendo dudas de los usuarios. Al concentrar y compartir conocimientos, estas comunidades ayudan a externalizar los esfuerzos de soporte técnico de los fabricantes. Es por esto que algunas de estas comunidades son creadas y mantenidas por los fabricantes originales (Jeppesen \& Frederiksen, 2006). En muchos casos estas comunidades también tienen un rol en la difusión de innovaciones creadas por los usuarios (Von Hippel, 2005). Este aspecto, la difusión, es clave dentro de la literatura sobre innovación por el usuario, la cual menciona dos métodos: distribución gratuita (Von Hippel, 2005) y comercialización (Bogers et. al., 2010) - cuando los usuarios se convierten en productores. En otros casos, las innovaciones son mantenidas en privado por sus creadores.

\section{Investigación}

El primer paso de la investigación fue construir un diagrama de flujo preliminar basado en los procesos descritos por la teoría de la innovación por el usuario para ser usado como herramienta de análisis de los distintos procesos y traspasos de información entre los actores de la innovación en BIM. El diagrama 1 muestra las posibles interacciones entre: tecnologías BIM estándares, usuarios e innovaciones generadas por los estos últimos. El flujo se inicia con (A) la introducción de las herramientas BIM a los usuarios. Luego, (B) dentro del grupo de usuarios surge un grupo menor llamado lead users o usuarios líderes, cuyas necesidades están adelantadas al mercado por lo cual tienden a innovar (Von Hippel, 1986). Esos usuarios en algunos casos (C) reciben apoyo directo de los productores de la tecnología, adicional al apoyo indirecto mencionado. A continuación (D, E), los usuarios líderes desarrollan sus innovaciones en un proceso iterativo de prueba y error que desarrollan en su propia práctica profesional. Finalmente (F), en algunos casos los usuarios líderes distribuyen su innovación de manera gratuita y $(G)$ en otros casos los usuarios líderes se convierten en productores al comercializar sus innovaciones. En línea segmentada se indican los flujos que no están necesariamente presentes en todos los casos.

Diagrama 1. Diagrama preliminar de la innovación por el usuario en BIM.

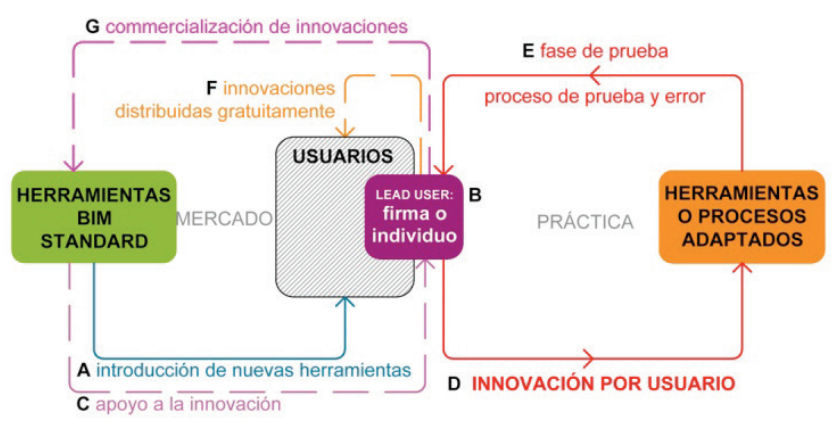

\section{Estudio de Casos}

Dado que la innovación por el usuario es un proceso informal del cual, generalmente, no existe literatura, la búsqueda preliminar de casos se realizó fundamentalmente a través de veinticinco entrevistas a usuarios y fabricantes de tecnologías BIM de distintos países. Los casos fueron seleccionados para ejemplificar diferentes posibilidades en relación a cuatro características observadas en la literatura acerca de innovación por el usuario: tipos de usuarios, motivaciones, apoyo directo de los fabricantes, y métodos de distribución de la innovación. Para mayor información sobre la investigación ver Soto, 2012.

Finalmente, se seleccionó ocho casos de distintos países que involucraban a distintos tipos de usuarios, Estos casos son: Revit Python Shell (RPS), Dynamo, goBIM, HOK Generador de muros cortina, ICABIM, MIT-CO Workshop, SOM BIM Dashbord y las plataformas de aplicaciones gratuitas BIM (PAG-BIM) ejemplificadas en cuatro casos: Case Apps, AEC Apps, Autodesk Exchange Apps y Revit Application Store. 


\section{Resultados}

Con respecto a las cuatro características observadas en la literatura los resultados para el caso de la innovación por el usuario en BIM se describen a continuación.

\section{Tipos de usuarios}

El estudio demostró que tanto individuos como empresas e instituciones sin fines de lucro están innovando para satisfacer sus necesidades específicas con respecto a las herramientas BIM. Esta diversidad de usuarios innovadores enriquece el proceso y crea oportunidades para el establecimiento de iniciativas de colaboración - por ejemplo, entre privados e instituciones académicas - que puede acelerar la tendencia a la innovación al promover la discusión y al combinar actores con distintos conocimientos y motivaciones.

\section{Motivaciones}

Los casos estudiados presentan dos tipos de motivaciones tras la innovación: una exploratoria, y otra de solución de problemas. Mientras las innovaciones de carácter exploratorio tienden a traspasar los límites de proyectos específicos, sólo en un caso la innovación generada para resolver un problema particular fue capaz de traspasar el ámbito del proyecto para el cual fue creada y ser aplicable de manera generalizada.

\section{Distribución de las innovaciones}

Al respecto, se presenta cierto patrón en el cual, mientras los individuos y las instituciones académicas tienden a distribuir sus innovaciones gratuitamente, las empresas privadas en cambio las mantienen solamente para uso interno. Dos de los casos estudiados presentaron comercialización.

\section{Apoyo directo de los fabricantes}

Sólo las innovaciones distribuidas gratuitamente obtuvieron apoyo directo de los fabricantes.

Adicionalmente, algunos temas específicos evidenciados a través del estudio son:

La innovación no está uniformemente distribuida. En algunos contextos de uso los usuarios son capaces de identificar las necesidades que no son satisfechas por las herramientas estándares, pero no tienen los recursos (habilidades de programación, dinero, apoyo de los fabricantes u otros) para innovar. En algunos de estos casos, y a modo de suplir la falta de recursos, los mismos distribuidores de las innovaciones generan algunas adaptaciones regionales para fomentar la adopción de sus productos.

Los kits de herramientas para el usuario no son suficientemente fáciles de usar. Este punto se relaciona directamente con el punto anterior, ya que debido a la complejidad de los kits sólo los usuarios con conocimiento avanzado de programación pueden innovar, dejando fuera de este proceso al usuario medio. Esto se observa en el caso de RPS, un plug-in para Revit y Vasari que permite programar en lenguaje Python dentro del ambiente BIM. En este caso su creador desarrolló una herramienta para facilitar la programación mejorando las capacidades de la API del programa, dado que incluso para él, un profesional del área de la programación, los kits de herramientas presentaban una excesiva complejidad de uso.

Existe una correlación entre el tipo de distribución de la innovación, el posible nivel de impacto de la ésta y la disponibilidad apoyo directo de los fabricantes. Este es el caso de RPS y Dynamo, ambas distribuidas por sus creadores como código abierto. Estas han recibido apoyo directo de los fabricantes del software original en la forma de apoyo directo en la programación - desarrolladores de Autodesk han trabajado en el código de las dos herramientas. El apoyo se ha traducido también en difusión en conferencias y blogs. Actualmente Dynamo, un plug-in para Autodesk Revit y Vasari que añade funcionalidades de programación visual a estos software, se distribuye gratuitamente a través de una plataforma online de Autodesk. Estos dos casos proponen un cambio drástico en la manera en que la herramienta original se utiliza e intentan facilitar el uso de los kits a usuarios con menor o nulo conocimiento de programación. Podemos inferir una correlación entre el potencial para impactar positivamente el uso - y cantidad de usuarios - de la tecnología original y la disponibilidad de apoyo por parte de sus fabricantes.

Las comunidades de usuarios pueden acelerar el progreso de la innovación. En casos como el de RPS, Dynamo y goBIM - esta última una aplicación que permite visualizar y anotar modelos Revit en dispositivos móviles - la distribución gratuita, o por un precio bajo, de una innovación fomenta su uso. Esto puede impulsar su desarrollo gracias al aumento de usuarios que testean y descubren problemas en el código, el efecto llamado Ley de Linus (Raymond, 1999).

Se intenta promover la creación colectiva, aunque aún de manera infructuosa. Los creadores de RPS y Dynamo declaran que una de sus motivaciones para lanzar sus herramientas como código abierto fue promover la colaboración de los usuarios en la programación de estas innovaciones, fomentando así la emergencia de creación colectiva (Allen, 1983). Sin embargo, ambos declararon haber obtenido cierta retroalimentación de los usuarios, pero no apoyo en la programación de la herramienta.

La distribución gratuita y/o como código abierto de las innovaciones reporta recompensas intrínsecas y extrínsecas a sus creadores. Algunas de las recompensas de liberar una herramienta como código abierto son la reciprocidad y el aumento de la reputación como programadores de sus creadores, lo que puede traducirse en nuevas oportunidades de trabajo. (Kogut \& Metiu, 2001; Von Hippel, 2005; Jeppesen \& Frederiksen, 2006) Este es el caso de los creadores de RPS y Dynamo quienes han visto su 
reputación aumentar e incluso han recibido ofertas laborales relacionadas con sus innovaciones.

La disponibilidad de plug-ins puede ayudar a resolver necesidades que se repiten a lo largo de ciertos contextos de uso sin que los usuarios necesiten innovar. Dado que las necesidades tienden a repetirse entre los usuarios, la aparición de comunidades en línea se torna un modo efectivo para los fabricantes de recopilar información acerca de esas necesidades repetitivas. Estas mismas plataformas sirven en algunos casos de base para la distribución de plug-ins que resuelven estas necesidades. En algunos casos estas comunidades son creadas y mantenidas por los fabricantes (Revit Application Store, Autodesk Exchange Apps) o por desarrolladores externos que usan la distribución gratuita o por precios bajos de estas aplicaciones como un medio de difusión de su trabajo (Case apps, AEC-apps). La inclusión en estas plataformas de sistemas de reseñas, así como la recopilación de aplicaciones creadas por los mismos usuarios ayudan a fomentar la idea de comunidad y la transferencia de información.

La participación de agentes externos ayuda a perfilar de mejor manera las necesidades y metas de los usuarios que se encuentran interesados en innovar. En los casos de las PAG-BIM y del MIT-CO Workshop se agrega un nuevo actor que complejiza las relaciones y traspasos de información e innovación del modelo original, dando acceso a la innovación a usuarios que no tienen los medios para innovar. El caso del MIT-CO Workshop se refiere al taller Laboratorio de Diseño Computacional: Reinventando BIM que fue realizado en MIT y financiado en parte por la oficina californiana de arquitectura CO. La idea detrás de la colaboración fue cuestionar las actuales capacidades de BIM y proponer innovaciones que transformaran o aumentaran las capacidades de la tecnología de manera creativa. En el caso del SOM BIM Dashboard - una plataforma para control de calidad que permite a SOM evaluar sus modelos BIM y extraer recomendaciones de buen uso que está siendo desarrollada pos SOM y Case - el agente externo ayuda además a delinear las reales necesidades de los usuarios asistiendo en el desarrollo de innovaciones más eficientes.

No todos los usuarios dentro de las firmas que innovan son usuarios líderes. La mayoría de los integrantes de una empresa son usuarios regulares sin la capacidad de innovar. Es por esto que las innovaciones dentro de las firmas deben ser fáciles de usar, generales - aplicables a más de un proyecto - deben estar bien documentadas, centralizadas y ser comunicadas por canales regulares a los usuarios. Si esto no es hecho de manera correcta se corre el riesgo de que las herramientas queden obsoletas y no sean utilizadas. HOK, una oficina de arquitectura que ha creado una serie de herramientas internas relacionadas con las tecnologías BIM, reconoce este potencial problema. Las herramientas deben ser utilizables no sólo por su desarrollador original y deben mantenerse vigentes incluso si éste ya no se encuentra en la firma.
Dentro de las firmas que innovan deben existir protocolos para enfrentar las necesidades de los usuarios. Este es el caso de ICABIM, un grupo dedicado al desarrollo de proyectos BIM dentro de la mayor empresa de ingeniería y construcción de México, ICA. Este grupo ha desarrollado aproximadamente sesenta aplicaciones para mejorar sus procesos BIM. Con el fin de identificar los requerimientos relevantes, necesarios de abordar a través de innovación, ICABIM ha establecido un mapa y sistemas de entrevistas y encuestas a modo de jerarquizar las necesidades de sus usuarios y abstraer innovaciones que puedan generar un alto impacto positivo en el trabajo del grupo. De esta manera, ICABIM se hace cargo de las características descritas en el punto anterior.

Es importante notar que estos protocolos deben usarse con precaución ya que pueden prevenir la exploración. En este sentido podríamos argumentar que innovaciones como Dynamo no pasarían los estándares de estos protocolos y nunca serían desarrolladas. Por otro lado, en grupos numerosos de usuarios las necesidades tenderán a ser variadas y heterogéneas, por lo cual deben existir mecanismos para su priorización.

\section{Conclusiones}

A través del estudio de casos la investigación demostró que existe innovación por parte de los usuarios en el caso de BIM. Usuarios provenientes de distintos contextos de uso están innovando principalmente a través de la creación de plug-ins para ingresar y extraer información de los modelos. Sin embargo, la investigación demostró que al contrario de lo indicado por Von Hippel (2005) a pesar de que existe una heterogeneidad de necesidades, la innovación para satisfacer estas necesidades no está uniformemente distribuida, ya que ésta depende de la disponibilidad de recursos que no son homogéneos entre los distintos contextos.

El estudio demuestra además que los traspasos de innovación e información descritos por la teoría original de innovación por el usuario, previstos dentro del diagrama de flujo uno, están presentes en la mayoría de los casos BIM. Adicionalmente, se integran nuevos agentes y flujos como se muestra en el diagrama dos.

En adición a los flujos originales (A-G) aparecen $(\mathrm{H})$ los agentes externos en la forma de desarrolladores (Case) u organizaciones de investigación (MIT), lo cual genera nuevos flujos como el ciclo de innovación compuesto por (I) el encargo de innovaciones a terceros o el trabajo colaborativo con estos y $(\mathrm{J})$ el proceso de comunicación de las innovaciones entre agente externo y usuarios líderes. Esto complejiza también los flujos de apoyo y distribución de la innovación, ya que se genera apoyo directo de los fabricantes (C) tanto a los usuarios líderes como a los agentes externos, y las innovaciones en algunos casos son (F) distribuidas gratuitamente por los usuarios líderes pero también por los agentes externos. Finalmente, ( $D$ y F) el proceso iterativo de desarrollo y testeo de la innovación ya no sólo sucede en las 
prácticas de los usuarios líderes sino que también en una práctica combinada entre éstos y los agentes externos.

Diagrama 2. Diagrama de flujo de la innovación por el usuario en BIM que resulta de superponer los diagramas de cada uno de los casos estudiados.

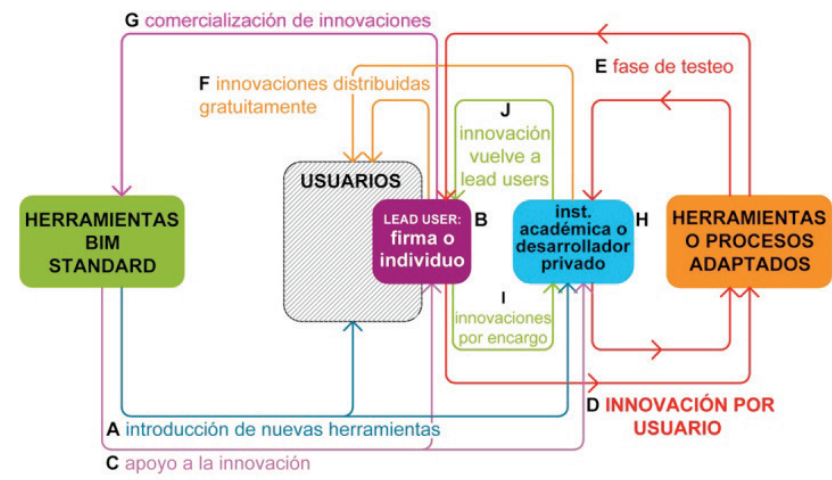

Se puede discutir que la aparición de un tercer agente invalida estos casos como reales innovaciones por el usuario, pero se incluyen dentro del estudio ya que en muchos casos su distribución gratuita permite satisfacer las necesidades de una gran cantidad de usuarios que no tiene los medios para innovar, permitiéndoles superar la complejidad de adaptación que presentan las herramientas BIM. Esto demuestra que si bien existe innovación por el usuario en el caso de BIM aún existe espacio de mejoras.

\section{Investigación Futura}

La investigación presentada en este artículo abre distintos caminos para exploraciones futuras. Un tema a investigar son los espacios de mejora para la adaptabilidad de las herramientas BIM. La investigación demostró que los kits de herramientas para usuarios aún son sólo utilizables por usuarios con alto conocimiento de programación, dejando fuera a una gran cantidad de usuarios medios. La posibilidad de abrir caminos para la innovación en BIM para usuarios sin conocimiento de programación implicaría una real democratización de la innovación en este ámbito. Otro camino que se abre como futura exploración es la búsqueda de nuevos casos de innovación asociados a distintos tipos de herramientas BIM, ya que las únicas innovaciones descubiertas dentro de la búsqueda preliminar fueron relacionadas al software BIM de un fabricante. Finalmente, otra área para una potencial exploración es el ámbito de la difusión de las innovaciones y cómo los usuarios medios se están beneficiando de las aplicaciones distribuidas gratuitamente por usuarios líderes, agentes externos y fabricantes.

\section{Referencias}

Allen, R. (1983). Collective Invention. Journal of Economic Behavior and Organization, 4,1-24

Bogers, M., Afuah, A., Bastian, B. (July 2010). Users as Innovators: A Review, Critique, and Future Research Directions. Journal of Management 36, (4), 857-875.

Jeppesen, L. B., \& Frederiksen, L. (January-February 2006). Why Do Users Contribute to Firm-Hosted User Communities? The Case of Computer-Controlled Music Instruments. Organization Science 17 (1), 45-63.

Kogut, B., Metiu, A. (2001). Open-source software development and distributed innovation. Oxford Review of Economic Policy 17 (2), 248264.

Lüthje, C., Herstatt, C., Von Hippel, E. (2005). User-Innovators and "Local" Information: The Case of Mountain Biking. Research Policy, 34, 965.

Maguire, M. (2001, October). Context of use Within Usability Activities. International Journal of Human-Computer Studies 55 (4), 453-483.

Polanyi, M. (1958). Personal Knowledge. Towards a Post-Critical Philosophy. Chicago: University of Chicago Press.

Raymond, E. (1999). The Cathedral and the Bazaar. Cambridge, Massachusetts: O’Reilly.

Soto, C. (2012). User Innovation in Digital Design and Construction: Dialectical Relations between Standard BIM Tools and Specific User Requirements. (Master Thesis). Massachusetts Institute of Technology. Retrieved from: http://dspace.mit.edu/bitstream/handle/1721.1/72975/808355734.p $\mathrm{df}$ ?sequence $=1$

Thomke, S. \& Von Hippel, E. (April 2002). Customers as Innovators. Harvard Business Review, 79-81.

Von Hippel, E. (1986, July). Lead Users: A Source of Novel Product Concepts. Management Science 32 (7), 791-805.

Von Hippel, E. (April 1994) "Sticky Information" and the Locus of Problem Solving: Implications for Innovation. Management Science 40, (4) 429-439.

Von Hippel, E. (2005). Democratizing Innovation. Cambridge:MIT Press. 